\title{
Prevalence of Self-Reported Hypertension and Antihypertensive Medication Use by County and Rural-Urban Classification — United States, 2017
}

\author{
Claudine M. Samanic, $\mathrm{PhD}^{1,2,3}$; Kamil E. Barbour, $\mathrm{PhD}^{1}$; Yong Liu, MD ${ }^{1}$; Yan Wang, $\mathrm{PhD}^{1}$; Jing Fang, MD ${ }^{4}$; Hua Lu, MS ${ }^{1}$; Linda Schieb, MSPH${ }^{4}$; \\ Kurt J. Greenlund, $\mathrm{PhD}^{1}$
}

In 2017, approximately one in three U.S. adults reported having been told by a health care professional that they had high blood pressure (hypertension) (1). Although hypertension prevalence is well documented at national and state levels, less is known about rural-urban variation and county-level prevalence. To examine prevalence of self-reported hypertension and antihypertensive medication use by rural-urban classification and county, CDC analyzed data reported by 442,641 adults aged $\geq 18$ years who participated in the 2017 Behavioral Risk Factor Surveillance System (BRFSS). In rural (noncore) areas, $40.0 \%$ (unadjusted prevalence) of adults reported having hypertension, whereas in the most urban (large central metro) areas, $29.4 \%$ reported having hypertension. Age-standardized hypertension prevalence was significantly higher in the most rural areas, compared with the most urban areas within nearly all categories of age, sex, and other demographic characteristics. Model-based hypertension prevalence across counties ranged from $18.0 \%$ to $55.0 \%$ and was highest in Southeastern* and Appalachian ${ }^{\dagger}$ counties. Model-based county-level prevalence of antihypertensive medication use among adults with hypertension ranged from $54.3 \%$ to $84.7 \%$. Medication use also was higher in rural areas compared with use in most urban areas, with prevalence highest in Southeastern and Appalachian counties as well as counties in the Dakotas and Nebraska. $\mathrm{CDC}$ is working with states to enhance hypertension awareness and management through a strategy of team-based care that involves physicians, nurses, pharmacists, dietitians, and community health workers. The increased use of telemedicine to support this strategy might improve access to care among underserved populations.

\footnotetext{
*https://worldpopulationreview.com/states/southeast-states.

$\dagger$ https://www.arc.gov/appalachian_region/TheAppalachianRegion.asp.
}

BRFSS $^{\circledR}$ is an annual, random-digit-dialed landline and mobile phone survey that is representative of the noninstitutionalized adult population aged $\geq 18$ years of the 50 states, the District of Columbia (DC), and U.S. territories. In 2017, 450,016 adults were interviewed, and data from 442,641 adults were included in this analysis. Data from 7,375 respondents were excluded because of incomplete survey responses or residence in U.S. territories (only data from residents of the 50 states and DC were included in this report). State-level response rates ranged from $30.6 \%$ to

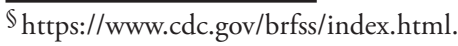

\section{INSIDE}

540 Improving Detection and Response to Respiratory Events - Kenya, April 2016-April 2020

545 Characteristics and Clinical Outcomes of Adult Patients Hospitalized with COVID-19 - Georgia, March 2020

551 Public Health Response to the Initiation and Spread of Pandemic COVID-19 in the United States, February 24-April 21, 2020

557 COVID-19 Among Workers in Meat and Poultry Processing Facilities - 19 States, April 2020

562 Notes from the Field: Large Measles Outbreak in Orthodox Jewish Communities - Jerusalem District, Israel, 2018-2019

564 QuickStats

Continuing Education examination available at https://www.cdc.gov/mmwr/mmwr_continuingEducation.html

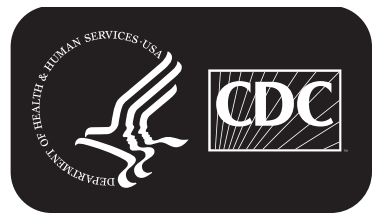

U.S. Department of Health and Human Services Centers for Disease Control and Prevention 
64.1\% (median $=45.9 \%$ ). Respondents were classified as having hypertension if they answered "yes" to the question "Have you ever been told by a doctor, nurse, or other health professional that you have high blood pressure?" Borderline and pregnancy-related hypertension were classified as "no." Respondents were classified as currently taking antihypertensive medication if they answered "yes" to the question "Are you currently taking medicine for your high blood pressure?" Those with "do not know" and missing data were excluded from analysis. All analyses, except for county-level estimates, applied sampling weights to account for the complex sample design, and data were weighted using an iterative proportional weighting (raking) procedure.**

Hypertension and antihypertensive medication use were examined by age group, sex, race/ethnicity, education, household income, and current health care coverage. Using the 2000 U.S. standard population (2), the age-standardized prevalence and $95 \%$ confidence intervals (CIs) for hypertension and antihypertensive medication use were estimated overall and by respondent characteristics including county rural-urban classification as defined by CDC's National Center for Health Statistics (large central metro/city, large fringe metro/suburb, medium metro, small metro, micropolitan, noncore/rural) (3).

\footnotetext{
Thtps://www.cdc.gov/brfss/annual_data/2017/pdf/2017-sdqr-508.pdf.

** https://www.cdc.gov/brfss/annual_data/2017/pdf/weighting-2017-508.pdf.
}

Unadjusted prevalences of hypertension and antihypertensive medication use at the county level was estimated using a multilevel regression model and poststratification approach (4) for 3,142 counties in all 50 states and DC. The multilevel logistic regression model for hypertension included self-reported data stratified by respondents' age group, sex, race/ethnicity, and other demographic characteristics from the 2017 BRFSS; county-level poverty data (percent below $150 \%$ of the federal poverty level) from the American Community Survey 5-year estimates (2013-2017); and random effects at county and state levels (group/aggregate variables). Model parameter estimates were applied to U.S. Census 2010 block-level population estimates by age, sex, and race/ethnicity to compute the predicted probability of having hypertension, and then generated the estimated prevalence at county-level through poststratification. A similar process was performed for antihypertensive medication use, except that the poststratification was conducted using only the population that reported having hypertension. The distribution of these county-level estimates is presented in quintiles. All analyses were conducted using SAS-callable SUDAAN (version 11.0.3; RTI International).

The unadjusted (age-standardized) prevalence of hypertension was $32.4 \%$ overall and increased consistently with increasing rurality, from $29.4 \%(28.5 \%)$ among persons living in large cities to $40.0 \%(34.1 \%)$ among those living in the most rural areas (Table 1). Age-specific hypertension prevalence was significantly higher in the most rural compared with

The MMWR series of publications is published by the Center for Surveillance, Epidemiology, and Laboratory Services, Centers for Disease Control and Prevention (CDC), U.S. Department of Health and Human Services, Atlanta, GA 30329-4027.

Suggested citation: [Author names; first three, then et al., if more than six.] [Report title]. MMWR Morb Mortal Wkly Rep 2020;69:[inclusive page numbers].

\section{Centers for Disease Control and Prevention}

Robert R. Redfield, MD, Director

Anne Schuchat, MD, Principal Deputy Director

Chesley L. Richards, MD, MPH, Deputy Director for Public Health Science and Surveillance

Rebecca Bunnell, PhD, MEd, Director, Office of Science

Arlene Greenspan, PhD, Acting Director, Office of Science Quality, Office of Science

Michael F. Iademarco, MD, MPH, Director, Center for Surveillance, Epidemiology, and Laboratory Services

MMWR Editorial and Production Staff (Weekly)

Charlotte K. Kent, PhD, MPH, Editor in Chief Jacqueline Gindler, MD, Editor

Paul Z. Siegel, MD, MPH, Guest Associate Editor

Mary Dott, MD, MPH, Online Editor

Terisa F. Rutledge, Managing Editor

Douglas W. Weatherwax, Lead Technical Writer-Editor

Glenn Damon, Soumya Dunworth, PhD, Teresa M. Hood, MS, Technical Writer-Editors

Michelle E. Bonds, MBA

Matthew L. Boulton, MD, MPH

Carolyn Brooks, ScD, MA

Jay C. Butler, MD

Virginia A. Caine, MD
Martha F. Boyd, Lead Visual Information Specialist Maureen A. Leahy, Julia C. Martinroe, Stephen R. Spriggs, Tong Yang, Visual Information Specialists

Quang M. Doan, MBA, Phyllis H. King,

Terraye M. Starr, Moua Yang,

Information Technology Specialists

MMWR Editorial Board

Timothy F. Jones, MD, Chairman

Katherine Lyon Daniel, PhD

Jonathan E. Fielding, MD, MPH, MBA

David W. Fleming, MD

William E. Halperin, MD, DrPH, MPH

Jewel Mullen, MD, MPH, MPA

Jeff Niederdeppe, $\mathrm{PhD}$

\author{
Patricia Quinlisk, MD, MPH \\ Patrick L. Remington, MD, MPH \\ Carlos Roig, MS, MA \\ William Schaffner, MD \\ Morgan Bobb Swanson, BS
}


TABLE 1. Unadjusted and age-standardized* prevalence of self-reported hypertension ${ }^{\dagger}$ among adults aged $\geq 18$ years, by urban-rural status ${ }^{\S}$ and selected characteristics - Behavioral Risk Factor Surveillance System, 2017

\begin{tabular}{|c|c|c|c|c|c|c|c|}
\hline Characteristic & Overall & $\begin{array}{l}\text { Large central } \\
\text { metro (city) }\end{array}$ & $\begin{array}{l}\text { Large fringe metro } \\
\text { (suburb) }\end{array}$ & Medium metro & Small metro & Micropolitan & Noncore (rural) \\
\hline No. of respondents & 442,641 & 70,197 & 84,608 & 92,346 & 61,579 & 66,711 & 67,200 \\
\hline Est. population $(x$ 1000) & 252,046 & 77,446 & 61,881 & 51,955 & 23,024 & 21,481 & 16,258 \\
\hline No. with hypertension & 178,312 & 25,446 & 32,969 & 36,761 & 25,098 & 28,196 & 29,842 \\
\hline $\begin{array}{l}\text { Est. population with } \\
\text { hypertension }(x \text { 1000) }\end{array}$ & 81,674 & 22,757 & 19,511 & 17,240 & 7,851 & 7,809 & 6,506 \\
\hline \multicolumn{8}{|l|}{ Prevalence, \% (95\% Cl) } \\
\hline Unadjusted & $32.4(32.1-32.7)$ & $29.4(28.7-30.0)$ & $31.5(31.0-32.1)$ & $33.2(32.6-33.7)$ & $34.1(33.4-34.8)$ & $36.4(35.6-37.1)$ & $40.0(39.1-40.9)$ \\
\hline Age-standardized* & $29.9(29.6-30.2)$ & $28.5(27.9-29.2)$ & $28.7(28.2-29.2)$ & $30.4(29.9-30.9)$ & $31.4(30.7-32.1)$ & $32.6(31.9-33.3)$ & $34.1(33.3-35.0)$ \\
\hline \multicolumn{8}{|l|}{ Age group (yrs), \% (95\% Cl) } \\
\hline $18-44^{* *}$ & $14.1(13.8-14.5)$ & $12.6(11.9-13.4)$ & $13.5(12.8-14.2)$ & $14.7(14.0-15.4)$ & $15.4(14.4-16.5)$ & $16.7(15.6-17.8)$ & $18.3(17.0-19.6)$ \\
\hline $45-64^{* *}$ & $40.5(40.0-41.0)$ & $39.5(38.2-40.7)$ & $38.0(37.0-39.0)$ & $40.5(39.6-41.4)$ & $43.1(41.8-44.3)$ & $44.6(43.3-45.8)$ & $46.1(44.7-47.5)$ \\
\hline$\geq 65^{* *}$ & $60.5(60.0-61.1)$ & $59.0(57.4-60.6)$ & $60.1(58.9-61.2)$ & $61.8(60.7-62.8)$ & $60.8(59.6-62.0)$ & $61.2(59.9-62.4)$ & $62.5(60.9-64.0)$ \\
\hline \multicolumn{8}{|l|}{ Sex, $* 0(95 \%$ Cl) } \\
\hline Male** & $32.9(32.5-33.3)$ & $30.8(29.9-31.7)$ & $31.9(31.1-32.7)$ & $33.7(32.9-34.4)$ & $34.8(33.7-35.9)$ & $35.9(34.8-37.0)$ & $37.4(36.1-38.7)$ \\
\hline Female ${ }^{* *}$ & $27.0(26.6-27.3)$ & $26.3(25.5-27.2)$ & $25.6(24.9-26.2)$ & $27.2(26.6-27.9)$ & $28.0(27.2-28.9)$ & $29.3(28.4-30.2)$ & $30.7(29.7-31.8)$ \\
\hline \multicolumn{8}{|l|}{ Race/Ethnicity,* \% (95\% Cl) } \\
\hline White, non-Hispanic ${ }^{* *}$ & $29.0(28.7-29.3)$ & $26.6(25.9-27.3)$ & $28.1(27.5-28.8)$ & $29.3(28.7-29.9)$ & $30.2(29.4-30.9)$ & $31.5(30.7-32.2)$ & $33.3(32.3-34.2)$ \\
\hline Black, non-Hispanic ${ }^{* *}$ & $40.0(39.2-40.9)$ & $39.1(37.6-40.6)$ & $36.6(34.9-38.4)$ & $41.8(40.3-43.4)$ & $43.5(40.4-46.7)$ & $47.8(44.5-51.1)$ & $46.1(43.1-49.2)$ \\
\hline Hispanic & $28.2(27.3-29.1)$ & $27.4(25.9-28.9)$ & $27.5(25.7-29.3)$ & $30.0(28.4-31.7)$ & $30.6(27.9-33.5)$ & $28.2(25.2-31.4)$ & $28.5(23.8-33.7)$ \\
\hline $\begin{array}{l}\text { American Indian/Alaska } \\
\text { Native, non-Hispanic }\end{array}$ & $37.1(34.7-39.5)$ & $37.7(30.3-45.7)$ & $35.2(30.1-40.7)$ & $35.4(31.5-39.6)$ & $36.8(32.6-41.3)$ & $38.7(34.8-42.9)$ & $38.1(34.2-42.2)$ \\
\hline Asian, non-Hispanic ${ }^{\dagger \dagger}$ & $23.8(21.9-25.8)$ & $22.5(19.5-25.8)$ & $25.9(22.7-29.3)$ & $24.5(21.8-27.4)$ & $19.5(15.1-24.9)$ & $26.9(22.2-32.0)$ & $37.4(24.3-52.7)$ \\
\hline $\begin{array}{l}\text { Native Hawaiian/Pacific } \\
\text { Islander, non-Hispanic }\end{array}$ & $33.0(28.3-38.0)$ & $26.0(18.2-35.8)$ & $39.8(33.4-46.6)$ & $40.2(33.6-47.1)$ & $30.3(21.7-40.5)$ & $35.3(26.5-45.2)$ & -\$§ \\
\hline $\begin{array}{l}\text { Multiracial, } \\
\text { non-Hispanic }{ }^{\dagger \dagger}\end{array}$ & $31.6(29.9-33.4)$ & $27.4(23.6-31.5)$ & $32.9(29.3-36.7)$ & $31.5(28.4-34.8)$ & $35.5(31.6-39.5)$ & $36.5(32.5-40.6)$ & $36.5(30.8-42.6)$ \\
\hline Other, non-Hispanic & $28.9(25.3-32.8)$ & $27.5(21.4-34.6)$ & $22.7(16.8-29.9)$ & $32.6(26.7-39.0)$ & $45.8(33.4-58.7)$ & $22.3(15.3-31.3)$ & $34.5(23.0-48.3)$ \\
\hline \multicolumn{8}{|l|}{ Education,* \% (95\% Cl) } \\
\hline Less than high school** & $35.4(34.4-36.3)$ & $32.6(30.7-34.6)$ & $35.7(33.6-37.8)$ & $37.1(35.3-38.9)$ & $36.0(33.8-38.2)$ & $36.9(34.4-39.5)$ & $39.4(36.7-42.1)$ \\
\hline $\begin{array}{l}\text { High school or } \\
\text { equivalent }\end{array}$ & $32.3(31.8-32.8)$ & $30.5(29.3-31.7)$ & $32.2(31.1-33.3)$ & $32.0(31.0-33.0)$ & $33.1(31.9-34.4)$ & $34.9(33.7-36.1)$ & $36.1(34.7-37.5)$ \\
\hline More than high school** & $27.5(27.2-27.8)$ & $26.8(26.1-27.6)$ & $26.3(25.7-26.8)$ & $28.0(27.5-28.6)$ & $29.2(28.3-30.2)$ & $29.8(28.9-30.7)$ & $30.8(29.7-31.9)$ \\
\hline \multicolumn{8}{|c|}{ Household income, $*$ \% (95\% Cl) } \\
\hline$<\$ 15,000^{+\dagger}$ & $37.9(36.9-39.0)$ & $35.1(33.0-37.3)$ & $40.5(38.2-42.7)$ & $38.4(36.7-40.2)$ & $38.1(35.7-40.7)$ & $40.3(37.8-42.9)$ & $41.8(38.7-45.0)$ \\
\hline$\$ 15,000$ to $<\$ 25,000^{* *}$ & $34.3(33.6-35.1)$ & $33.0(31.4-34.6)$ & $33.4(31.7-35.1)$ & $34.0(32.6-35.4)$ & $37.6(35.8-39.4)$ & $36.2(34.4-38.1)$ & $36.8(35.0-38.7)$ \\
\hline$\$ 25,000$ to $<\$ 35,000^{* *}$ & $31.9(30.9-32.9)$ & $30.8(28.5-33.3)$ & $29.7(27.8-31.8)$ & $32.6(30.9-34.4)$ & $31.2(29.2-33.2)$ & $35.9(33.4-38.5)$ & $36.3(33.7-39.0)$ \\
\hline$\$ 35,000$ to $<\$ 50,000^{* *}$ & $29.9(29.1-30.7)$ & $27.9(26.1-29.8)$ & $28.7(27.2-30.3)$ & $31.4(30.0-32.9)$ & $32.6(30.6-34.6)$ & $31.0(29.2-32.8)$ & $32.4(30.0-34.8)$ \\
\hline$\geq \$ 50,000^{\dagger \dagger}$ & $26.9(26.5-27.3)$ & $25.7(24.8-26.6)$ & $26.5(25.7-27.2)$ & $27.1(26.3-27.8)$ & $28.0(26.8-29.3)$ & $29.4(28.2-30.5)$ & $31.0(29.5-32.5)$ \\
\hline \multicolumn{8}{|c|}{ Health care coverage, ${ }^{*} \%(95 \% \mathrm{Cl})$} \\
\hline Yes $^{* *}$ & $30.1(29.8-30.4)$ & $28.8(28.2-29.5)$ & $28.8(28.2-29.3)$ & $30.6(30.0-31.1)$ & $31.6(30.8-32.3)$ & $33.0(32.3-33.8)$ & $34.8(33.9-35.7)$ \\
\hline $\mathrm{No}^{* *}$ & $27.5(26.3-28.7)$ & $25.0(22.6-27.6)$ & $27.2(24.8-29.8)$ & $29.4(27.2-31.6)$ & $30.2(27.7-32.9)$ & $28.2(26.0-30.6)$ & $30.6(27.7-33.6)$ \\
\hline
\end{tabular}

Abbreviation: $\mathrm{Cl}=$ confidence interval.

${ }^{*}$ All estimates, with the exception of age-group estimates, were age-standardized to the 2000 U.S standard population aged $\geq 18$ years using three age groups $(18-44,45-64$, and $\geq 65$ years).

† Hypertension was defined as an affirmative response to"Have you ever been told by a doctor, nurse, or other health professional that you have high blood pressure?"

Preeclampsia, borderline high, or prehypertensive was categorized as "no."

$\S$ County urbanization levels were determined using the 2013 National Center for Health Statistics Urban-Rural Classification Scheme for Counties. https://www.cdc. gov/nchs/data/series/sr_02/sr02_166.pdf.

" Weighted number of adults in the population with hypertension.

** Age-standardized prevalence significantly higher in the most rural (noncore) compared with the most urban (large central metro) areas at $p<0.01$.

${ }^{\dagger+}$ Age-standardized prevalence significantly higher in the most rural (noncore) compared with the most urban (large central metro) areas at $p<0.05$.

$\S \S$ Estimates are unreliable and are suppressed (sample size $<50$ or relative standard error $>30 \%$ ).

the most urban areas for each age group. Age-standardized hypertension prevalence was significantly higher in the most rural compared with the most urban areas for men, women, non-Hispanic whites, non-Hispanic blacks, non-Hispanic Asians, non-Hispanic multiracial adults, all levels of education and income, and among respondents with or without current health care coverage. Among those with hypertension, the unadjusted (age-standardized) percentage of those currently taking antihypertensive medication ranged from $73.0 \%$ $(56.2 \%)$ to $80.2 \%(64.8 \%)$ (Table 2). Age-specific prevalence 
Morbidity and Mortality Weekly Report

TABLE 2. Unadjusted and age-standardized* prevalence of current antihypertensive medication use ${ }^{\dagger}$ among adults aged $\geq 18$ years reporting hypertension, by urban-rural status ${ }^{\S}$ and selected characteristics - Behavioral Risk Factor Surveillance System, 2017

\begin{tabular}{|c|c|c|c|c|c|c|c|}
\hline Characteristic & Overall & $\begin{array}{l}\text { Large central } \\
\text { metro (city) }\end{array}$ & $\begin{array}{l}\text { Large fringe } \\
\text { metro (suburb) }\end{array}$ & Medium metro & Small metro & Micropolitan & Noncore (rural) \\
\hline No. of respondents & 178,312 & 25,446 & 32,969 & 36,761 & 25,098 & 28,196 & 29,842 \\
\hline Est. population (x 1000) & 81,527 & 22,728 & 19,481 & 17,209 & 7,836 & 7,780 & 6,492 \\
\hline $\begin{array}{l}\text { No. using antihypertensive } \\
\text { medication }\end{array}$ & 146,754 & 20,422 & 27,171 & 30,286 & 20,652 & 23,291 & 24,932 \\
\hline $\begin{array}{l}\text { Est. population using } \\
\text { antihypertensive medication } \\
(x 1000)^{9}\end{array}$ & 61,927 & 16,586 & 14,886 & 13,219 & 5,963 & 6,063 & 5,210 \\
\hline \multicolumn{8}{|l|}{ Prevalence, \% (95\% CI) } \\
\hline Unadjusted & $76.0(75.5-76.4)$ & $73.0(71.7-74.2)$ & $76.4(75.4-77.4)$ & 76.8 (75.9-77.7) & $76.1(74.9-77.3)$ & $77.9(76.8-79.0)$ & $80.2(79.1-81.4)$ \\
\hline Age-standardized* & $59.6(58.8-60.3)$ & $56.2(54.6-57.9)$ & $59.7(58.2-61.2)$ & $60.8(59.4-62.1)$ & $60.2(58.2-62.2)$ & $62.6(60.6-64.5)$ & $64.8(62.6-66.9)$ \\
\hline \multicolumn{8}{|l|}{ Age group (yrs), \% (95\% Cl) } \\
\hline $18-44^{* *}$ & $37.9(36.5-39.2)$ & $32.7(29.9-35.7)$ & $38.2(35.5-40.9)$ & $39.5(37.0-42.1)$ & $38.8(35.2-42.6)$ & $42.5(39.0-46.0)$ & $46.2(42.3-50.1)$ \\
\hline $45-64^{* *}$ & 79.6 (78.9-80.3) & $77.8(76.0-79.4)$ & $79.4(78.0-80.7)$ & 80.7 (79.5-81.9) & $79.8(78.1-81.4)$ & $81.4(79.9-82.7)$ & $82.1(80.5-83.7)$ \\
\hline$\geq 65$ & $92.0(91.5-92.4)$ & $91.7(90.6-92.8)$ & $92.0(91.0-92.8)$ & $91.9(91.1-92.6)$ & $92.3(91.4-93.1)$ & $92.1(90.8-93.3)$ & $92.4(91.5-93.2)$ \\
\hline \multicolumn{8}{|l|}{ Sex, ${ }^{*} \%(95 \% \mathrm{Cl})$} \\
\hline Male $e^{* *}$ & $56.7(55.8-57.6)$ & $52.4(50.5-54.3)$ & $57.5(55.6-59.3)$ & $58.1(56.3-59.9)$ & $57.8(55.0-60.5)$ & $59.5(57.1-61.9)$ & $61.5(58.7-64.3)$ \\
\hline Female** & $64.0(62.7-65.2)$ & $61.6(58.7-64.3)$ & $63.2(60.7-65.6)$ & $64.8(62.6-67.0)$ & $64.1(61.2-66.9)$ & $67.5(64.2-70.6)$ & $69.7(66.4-72.8)$ \\
\hline \multicolumn{8}{|l|}{ Race/Ethnicity,* \% (95\% CI) } \\
\hline White, non-Hispanic** & $59.0(58.1-59.9)$ & $53.7(51.6-55.8)$ & $58.9(57.0-60.8)$ & $60.4(58.6-62.2)$ & $59.4(57.2-61.5)$ & $60.5(58.4-62.5)$ & $64.8(62.4-67.0)$ \\
\hline Black, non-Hispanic & $68.1(66.2-70.0)$ & $65.1(61.7-68.3)$ & $66.4(62.3-70.3)$ & $69.9(66.5-73.1)$ & $72.4(65.4-78.5)$ & $77.4(70.8-82.8)$ & $71.3(65.0-76.8)$ \\
\hline Hispanic ${ }^{\dagger \dagger}$ & $54.0(51.9-56.0)$ & $51.5(48.1-55.0)$ & $55.1(50.8-59.3)$ & $54.6(51.0-58.0)$ & $55.8(50.0-61.4)$ & $61.1(53.7-68.0)$ & $65.1(51.8-76.5)$ \\
\hline $\begin{array}{l}\text { American Indian/Alaska Native, } \\
\text { non-Hispanic }\end{array}$ & $58.6(53.6-63.5)$ & $59.2(44.9-72.1)$ & $56.1(43.6-67.9)$ & $61.6(52.1-70.3)$ & $57.9(46.7-68.4)$ & $57.0(49.1-64.5)$ & $57.8(50.5-64.7)$ \\
\hline Asian, non-Hispanic & $58.0(52.8-63.0)$ & $55.9(47.4-64.0)$ & $61.7(54.2-68.6)$ & $64.5(53.9-73.9)$ & $40.9(33.3-48.9)$ & $61.4(46.4-74.6)$ & $47.1(36.3-58.1)$ \\
\hline $\begin{array}{l}\text { Native Hawaiian/Pacific Islander, } \\
\text { non-Hispanic }\end{array}$ & $54.9(45.8-63.6)$ & —§§ & —§§ & $53.2(38.0-67.7)$ & —§§ & —§§ & —§§ \\
\hline Multiracial, non-Hispanic & $56.7(52.8-60.6)$ & $62.9(53.0-71.9)$ & $51.0(45.0-57.0)$ & $57.9(51.4-64.0)$ & $49.3(41.2-57.5)$ & $54.6(47.6-61.4)$ & $52.6(43.7-61.3)$ \\
\hline Other, non-Hispanic & $54.9(45.4-64.0)$ & $49.8(35.0-64.6)$ & $47.7(37.7-57.7)$ & $66.2(48.1-80.5)$ & $70.2(43.4-87.9)$ & $38.7(25.1-54.2)$ & $44.8(25.4-65.9)$ \\
\hline \multicolumn{8}{|l|}{ Education, ${ }^{*} \%(95 \% \mathrm{Cl})$} \\
\hline Less than high school ${ }^{\dagger \dagger}$ & $58.6(56.4-60.8)$ & $55.1(50.4-59.7)$ & $58.3(53.5-62.8)$ & $60.0(55.7-64.3)$ & $56.3(51.4-61.0)$ & $65.5(59.1-71.4)$ & $64.4(58.1-70.2)$ \\
\hline High school or equivalent** & $59.6(58.4-60.9)$ & $56.8(53.7-59.9)$ & $58.8(55.9-61.6)$ & $59.9(57.4-62.4)$ & $60.7(57.3-63.9)$ & $62.2(59.2-65.1)$ & $64.4(61.2-67.5)$ \\
\hline More than high school** & $59.8(58.8-60.8)$ & $56.4(54.4-58.5)$ & $60.5(58.6-62.4)$ & $61.5(59.7-63.2)$ & $60.9(57.9-63.8)$ & $61.8(59.2-64.3)$ & $65.4(62.2-68.5)$ \\
\hline \multicolumn{8}{|l|}{ Household income, $* \%(95 \% \mathrm{Cl})$} \\
\hline$<\$ 15,000$ & $61.5(59.3-63.7)$ & $58.1(53.5-62.4)$ & $63.2(58.2-67.9)$ & $63.5(59.5-67.5)$ & $58.8(53.6-63.8)$ & $65.0(58.8-70.8)$ & $64.6(58.2-70.6)$ \\
\hline$\$ 15,000$ to $<\$ 25,000^{\dagger \dagger}$ & $59.7(57.9-61.5)$ & $54.6(50.7-58.3)$ & $59.5(55.1-63.7)$ & $60.4(57.0-63.8)$ & $62.6(58.0-66.9)$ & $65.7(61.1-69.9)$ & $66.4(61.7-70.8)$ \\
\hline$\$ 25,000$ to $<\$ 35,000$ & $60.4(57.5-63.2)$ & $58.7(51.7-65.3)$ & $62.2(56.7-67.3)$ & $60.1(55.1-64.8)$ & $60.5(54.8-65.9)$ & $60.5(54.2-66.5)$ & $62.8(57.1-68.2)$ \\
\hline$\$ 35,000$ to $<\$ 50,000$ & $56.9(54.9-58.8)$ & $56.6(51.5-61.6)$ & $55.8(51.7-59.9)$ & $57.0(53.5-60.4)$ & $52.8(49.0-56.5)$ & $60.8(56.1-65.3)$ & $61.8(56.4-66.9)$ \\
\hline$\geq \$ 50,000^{\dagger \dagger}$ & $59.7(58.5-60.9)$ & $56.0(53.4-58.4)$ & $59.7(57.4-61.9)$ & $61.7(59.3-64.0)$ & $61.9(57.8-65.8)$ & $61.2(58.0-64.3)$ & $65.7(61.5-69.7)$ \\
\hline \multicolumn{8}{|l|}{ Health care coverage, $* \%(95 \% \mathrm{CI})$} \\
\hline Yes** & $61.6(60.8-62.4)$ & $58.6(56.8-60.4)$ & $61.0(59.4-62.6)$ & $62.8(61.3-64.3)$ & $62.3(60.1-64.5)$ & $64.3(62.2-66.4)$ & $67.7(65.4-69.9)$ \\
\hline No & $47.2(45.3-49.1)$ & $43.3(39.8-46.8)$ & $50.3(45.9-54.7)$ & $49.0(45.2-52.8)$ & $44.5(38.7-50.5)$ & $52.1(47.4-56.8)$ & $48.5(43.5-53.5)$ \\
\hline
\end{tabular}

Abbreviation: $\mathrm{Cl}=$ confidence interval.

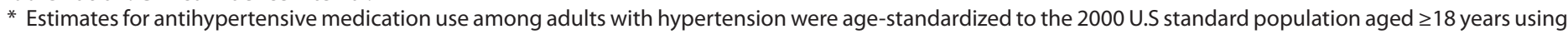
three age groups (18-44, 45-64, and $\geq 65$ years).

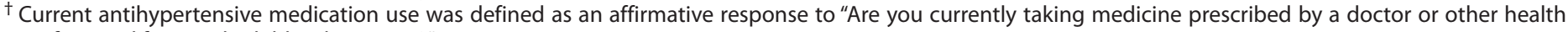
professional for your high blood pressure?"

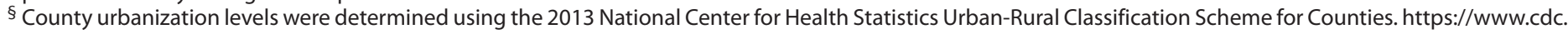
gov/nchs/data/series/sr_02/sr02_166.pdf.

१ Weighted number of adults in the population currently using antihypertensive medication.

** Age-standardized prevalence significantly higher in the most rural (noncore) compared with the most urban (large central metro) areas at $p<0.01$.

t+ Age-standardized prevalence significantly higher in the most rural (noncore) compared with the most urban (large central metro) areas at $\mathrm{p}<0.05$.

$\S \S$ Estimates are unreliable and are suppressed (sample size $<50$ or relative standard error $>30 \%$ ).

of current medication use among persons reporting hypertension was significantly higher in the most rural compared with the most urban areas for respondents aged $<65$ years, but similar for those aged $\geq 65$ years. Age-standardized prevalence of medication use was significantly higher in the most rural compared with the most urban areas for men, women, nonHispanic whites, Hispanic adults, all levels of education, and among respondents with current health care coverage. In each rural-urban category, hypertension prevalence was higher among men than women, but among adults with hypertension, 
prevalence of medication use was higher among women than among men.

County-level predicted hypertension prevalence ranged from $18.0 \%$ to $55.0 \%$ (Figure). The majority of counties in the Southeast and Appalachia were in the highest quintile, which is consistent with the rurality of most of the counties in these regions. Among persons reporting hypertension, the predicted prevalence of antihypertensive medication use ranged from $54.3 \%$ to $84.7 \%$. Counties in the Southeast, Appalachia, and Great Plains ${ }^{\dagger \dagger}$ were in the highest quintile for current medication use among adults with hypertension. Within the Southeastern states with high hypertension prevalences, estimated prevalences of medication use varied widely across counties.

\section{Discussion}

This report provides the most recent data on self-reported prevalence of diagnosed hypertension and antihypertensive medication use. Geographic variability was evident by both rural-urban status and at the county level using model-based estimates. Results suggest that as many as one in two adults in some counties might have hypertension.

Rural populations in the United States have a higher prevalence of many chronic conditions and risk factors (5) and experience disparities in access to care such as limited access to health care personnel and lack of public transportation (G). Results from studies examining the prevalence of risk factors for hypertension and other cardiovascular diseases highlight that prevalences of obesity (7), cigarette smoking (5), and physical inactivity (5) are higher in rural areas. Rural communities might also be more affected by poor access to affordable healthy food options (8).

Age-standardized hypertension prevalence was $28.5 \%$ in the most urban and $34.1 \%$ in the most rural areas, respectively. This is consistent with data from the 2013 BRFSS, which showed that respondents in nonmetropolitan counties were more likely to report hypertension $(38.1 \%)$ than were those in metropolitan counties (32.6\%) (9). Those data also showed that hypertension prevalence decreased as county economic status improved for both metropolitan and nonmetropolitan counties. However, within every level of county economic status, hypertension prevalence was lower in metropolitan counties than in nonmetropolitan counties (9). In the present study, hypertension prevalence was higher in the most rural compared with the most urban areas within every level of household income.

\begin{abstract}
Summary
What is already known about this topic?

Prevalence of hypertension increases with age, is higher among men and among non-Hispanic blacks, and has been consistently higher in the Southeastern region of the United States.

What is added by this report?

The unadjusted prevalence of hypertension was $40.0 \%$ in the most rural areas and $29.4 \%$ in the most urban areas. Countylevel prevalence of hypertension ranged from $18.0 \%$ to $55.0 \%$ (highest in the Southeast and Appalachia). County-level prevalence of antihypertensive medication use (among persons reporting hypertension) ranged from $54.4 \%$ to $84.7 \%$ (highest in the Southeast).

What are the implications for public health practice?

$\mathrm{CDC}$ is working with states to improve hypertension treatment and control through team-based care interventions that include the increased use of telemedicine.
\end{abstract}

Antihypertensive medication use prevalence overall was higher in older age groups and highest among non-Hispanic blacks in each category of rural-urban classification, consistent with the higher prevalence of hypertension observed in these subgroups. Differences in prevalence of medication use by urban-rural status decreased with increasing age, and prevalence was similar across all urban-rural categories for those aged $\geq 65$ years. Prevalence of medication use was higher among women despite the higher prevalence of hypertension among men. This overall gender difference has been reported elsewhere (1), but the reasons for it are unclear. Data from Medicare Part $\mathrm{D}$ beneficiaries aged $\geq 65$ years suggest that antihypertensive medication nonadherence is similar for men (25.8\%) and women $(26.7 \%)$ (10). In addition to counties in the Southeast and Appalachia, prevalence of antihypertensive medication use among persons with self-reported hypertension was also highest in Nebraska and the Dakotas, despite a relatively lower prevalence of hypertension in these states. Medication use is the most important intervention to control hypertension, although lifestyle interventions can be adopted among those with stage 1 hypertension (blood pressure range $=130-139 / 80-89 \mathrm{mmHg}$ ) with low estimated cardiovascular risk. ${ }^{\$ \$}$ More information is needed to understand variation in antihypertensive medication use prevalence, such as the percentage of persons who choose to adopt lifestyle changes in lieu of medication and how this might vary by age, gender, and urban-rural status.

\footnotetext{
$\$ \$$ https://www.acc.org/ /media/Non-Clinical/Files-PDFs-Excel-MS-Word-etc/ Guidelines/2017/Guidelines_Made_Simple_2017_HBP.pdf.
}

\footnotetext{
${ }_{\dagger \dagger}^{\dagger}$ https://www.newworldencyclopedia.org/entry/Great_Plains.
} 
FIGURE. Model-based prevalence of self-reported hypertension (A) and antihypertensive medication use (B) among adults aged $\geq 18$ years, by county - Behavioral Risk Factor Surveillance System, 2017*

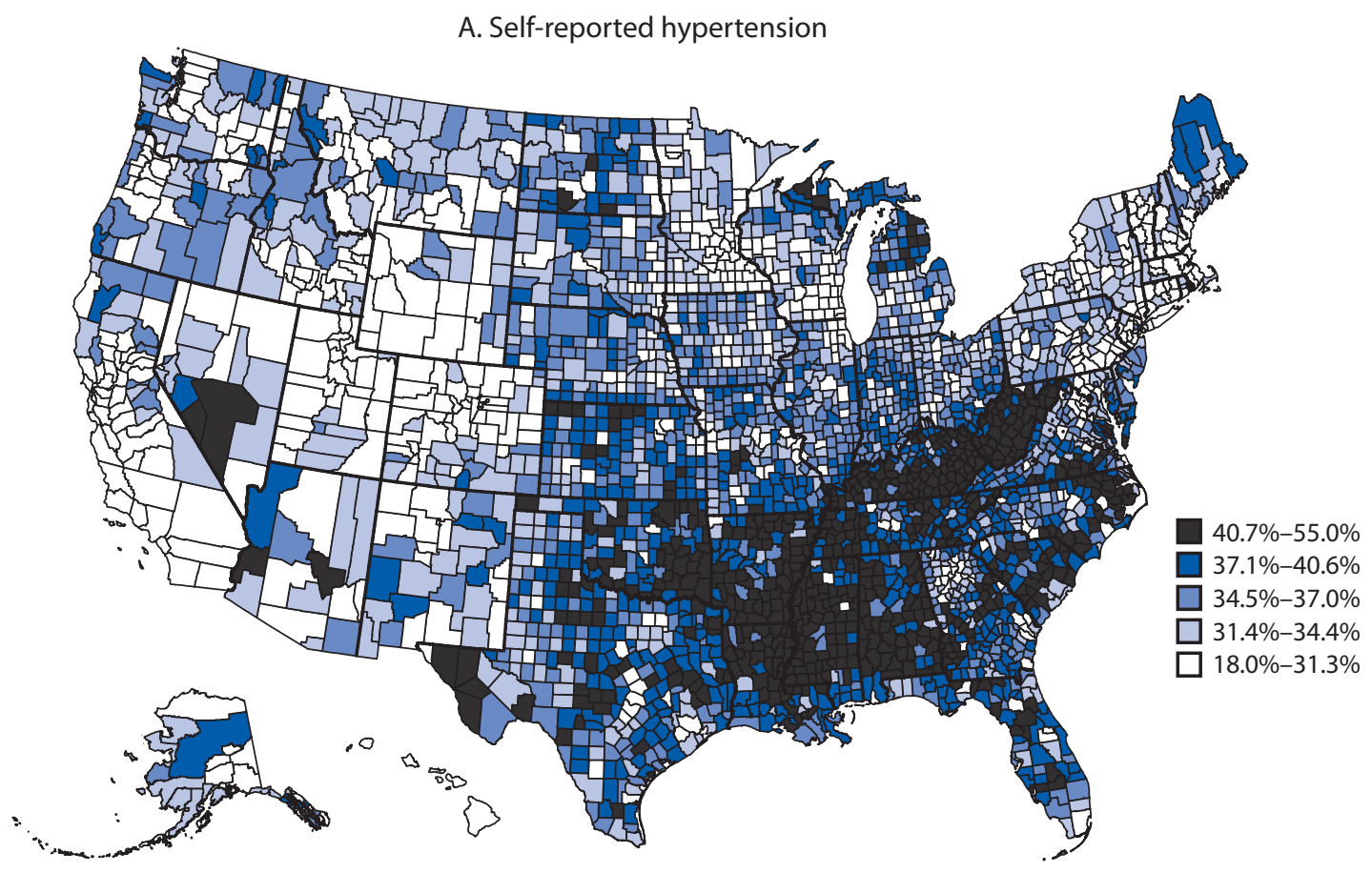

B. Self-reported antihypertensive medication use among adults with hypertension

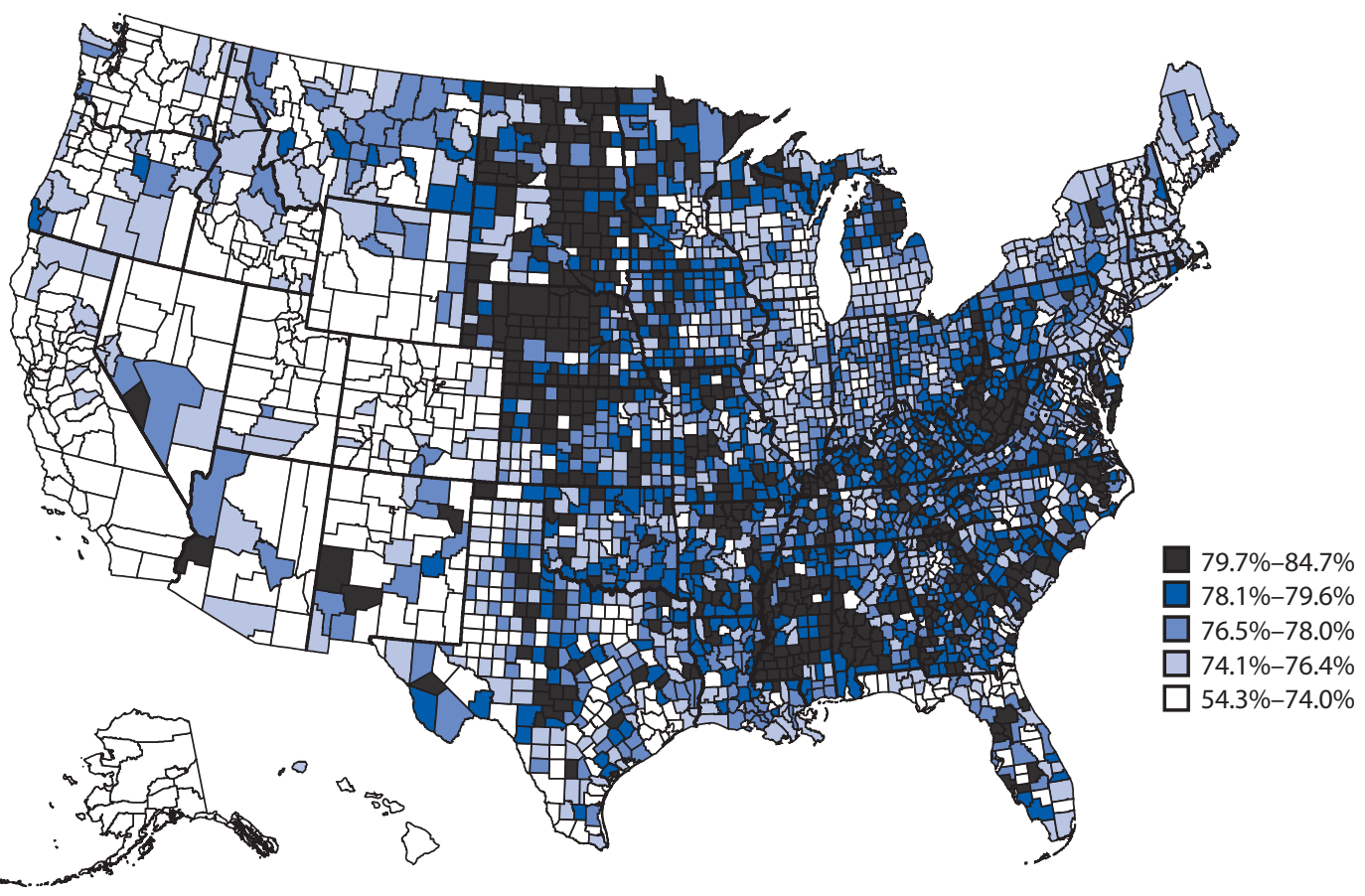

* Map A includes the 442,641 respondents to the 2017 Behavioral Risk Factor Surveillance System; Map B is limited to the 178,312 respondents with hypertension. 
The findings in this report are subject to at least three limitations. First, results are based on self-reported data and might or might not reflect hypertension estimates based on clinical measurements of blood pressure. Second, low median response rates might limit the representativeness of the 2017 BRFSS sample, potentially resulting in either under- or overestimates of prevalence, although application of sampling weights is likely to reduce the impact of some of the nonresponse bias on the overall estimates. Finally, county-level prevalence was estimated via small area estimation, and the modeling process could introduce bias. The validation and limitations of this methodology have been fully discussed (4).

Hypertension is a major risk factor for cardiovascular disease and is a substantial public health concern. CDC is working with states to improve hypertension treatment and control through team-based care interventions that involve physicians, nurses, pharmacists, dietitians, and community health workers. The increased use of telemedicine to support this strategy might improve the quality and availability of care among underserved populations.

Corresponding author: Kamil E. Barbour, iyk1@cdc.gov, 770-488-5145.

\footnotetext{
${ }^{1}$ Division of Population Health, National Center for Chronic Disease Prevention and Health Promotion, CDC; ${ }^{2}$ Indiana State Department of Health; ${ }^{3}$ Moffitt Cancer Center, Tampa, Florida; ${ }^{4}$ Division for Heart Disease and Stroke Prevention, National Center for Chronic Disease Prevention and Health, CDC.

All authors have completed and submitted the International Committee of Medical Journal Editors form for disclosure of potential conflicts of interest. No potential conflicts of interest were disclosed.
}

\section{References}

1. Samanic CM, Barbour KE, Liu Y, Fang J, Lu H, Schieb L, Greenlund KJ. Prevalence of self-reported hypertension and antihypertensive medication use among adults-United States, 2017. MMWR Morb Mortal Wkly Rep 2020;69:393-8. http://dx.doi.org/10.15585/mmwr.mm6914a1

2. Klein RJ, Schoenborn CA. Age adjustment using the 2000 projected U.S. population. Healthy people statistical notes, no. 20. Hyattsville, MD: US Department of Health and Human Services, CDC, National Center for Health Statistics; 2001. https://www.cdc.gov/nchs/data/ statnt/statnt20.pdf

3. Ingram DD, Franco SJ. 2013 NCHS urban-rural classification scheme for counties. Vital Health Stat 2 2014;166:1-73.

4. Zhang X, Holt JB, Yun S, Lu H, Greenlund KJ, Croft JB. Validation of multilevel regression and poststratification methodology for small area estimation of health indicators from the behavioral risk factor surveillance system. Am J Epidemiol 2015;182:127-37. https://doi.org/10.1093/ aje/kwv002

5. Matthews KA, Croft JB, Liu Y, et al. Health-related behaviors by urbanrural county classification-United States, 2013. MMWR Surveill Summ 2017;66(No. SS-5). https://doi.org/10.15585/mmwr.ss6605a1

6. Douthit N, Kiv S, Dwolatzky T, Biswas S. Exposing some important barriers to health care access in the rural USA. Public Health 2015;129:611-20. https://doi.org/10.1016/j.puhe.2015.04.001

7. Lundeen EA, Park S, Pan L, O'Toole T, Matthews K, Blanck HM. Obesity prevalence among adults living in metropolitan and nonmetropolitan counties-United States, 2016. MMWR Morb Mortal Wkly Rep 2018;67:653-8. https://doi.org/10.15585/mmwr.mm6723a1

8. Larson NI, Story MT, Nelson MC. Neighborhood environments: disparities in access to healthy foods in the U.S. Am J Prev Med 2009;36:74-81.e10. https://doi.org/10.1016/j.amepre.2008.09.025

9. Shaw KM, Theis KA, Self-Brown S, Roblin DW, Barker L. Chronic disease disparities by county economic status and metropolitan classification, Behavioral Risk Factor Surveillance System, 2013. Prev Chronic Dis 2016;13:160088. https://doi.org/10.5888/pcd13.160088

10. Ritchey M, Chang A, Powers C, et al. Vital signs: disparities in antihypertensive medication nonadherence among Medicare Part D beneficiaries-United States, 2014. MMWR Morb Mortal Wkly Rep 2016;65:967-76. https://doi.org/10.15585/mmwr.mm6536e1 